\title{
Size, shape, and stability of InAs quantum dots on the GaAs(001) substrate
}

\author{
L. G. Wang, ${ }^{*}$ P. Kratzer, N. Moll, and M. Scheffler \\ Fritz-Haber-Institut der Max-Planck-Gesellschaft, Faradayweg 4-6, D-14195 Berlin-Dahlem, Germany
}

(Received 18 June 1999; revised manuscript received 21 January 2000)

\begin{abstract}
We study the energetics of island formation in Stranski-Krastanow growth of highly mismatched heteroepitaxy within a parameter-free approach. It is shown that the (frequently found) rather narrow size distribution of the self-assembled coherent islands can be understood as the result of the system being trapped in a constrained equilibrium state with a fixed island density. If allowing for variations of the island density, we find that larger islands combined with a lower island density are more stable; this implies that Ostwald ripening will take place on time scales sufficiently long for exchange of atoms between different islands to occur. Moreover, we show how to select the island size by controlling the growth conditions and the amount of deposited material. Our study also indicates that the island shape depends on the island size, i.e., an island with larger volume has a higher value of the height-to-base ratio.
\end{abstract}

\section{INTRODUCTION}

In recent years, the surface morphology of overlayers in heteroepitaxial growth has attracted much interest. One of the driving forces behind this development is the goal to create nanostructures by exploiting the effect of selforganization during growth. Such nanostructures, apart from being an interesting object of basic research, have opened up a developing field of applications, mainly in optoelectronic devices. In thin film deposition, traditionally three different growth modes have been distinguished on phenomenological grounds, namely, layer-by-layer or two-dimensional (2D) growth (the so-called Frank-Van der Merwe mode ${ }^{1}$ ), island (3D) growth (the so-called Volmer-Weber mode ${ }^{2}$ ), and first 2D growth of a few monolayers (MLs) thick wetting layer followed by 3D growth (referred to as the StranskiKrastanow mode ${ }^{3}$ ). As early as 1958 , a classification of these growth modes on the basis of thermodynamics has been developed. Following Bauer, ${ }^{4}$ the growth mode of the film is determined by the relative weight of the free enthalpy of the substrate surface $\Gamma_{s}$ of the surface of the film $\Gamma_{f}$ and of the interface between the film and the substrate $\Gamma_{i}$. If $\Delta \Gamma=\Gamma_{f}$ $+\Gamma_{i}-\Gamma_{s} \leqslant 0$ is fulfilled independently of the film thickness (for homoepitaxial growth $\Delta \Gamma=0$ ), then this theory predicts Frank-Van der Merwe growth. If $\Delta \Gamma>0$, Volmer-Weber growth is expected. In many cases, $\Delta \Gamma$ is found to be negative for the first few monolayers, and later becomes positive beyond a critical layer thickness. This results in StranskiKrastanow growth. In particular, this situation occurs frequently in heteroepitaxial growth, where the material of the deposited film has a different bulk lattice constant than the substrate material. The terms $\Gamma_{f}$ and $\Gamma_{i}$ contain contributions from the elastic energy due to the strain introduced by the lattice mismatch, and the film will therefore become unstable when it exceeds a certain critical thickness. However, one should keep in mind that a rough surface morphology may also occur for reasons other than thermodynamics. For instance, thermodynamic equilibrium may not be reached because of sizeable diffusion barriers and/or Schwoebel barriers, resulting in a rough surface similar to StranskiKrastanow growth, even in cases where Frank-Van der
Merwe growth is predicted by thermodynamics. In these cases, film growth is dominated by the kinetics of the deposition process. ${ }^{5-7}$

In this article, we deal with the heteroepitaxial growth of InAs on GaAs. Due to the large lattice mismatch of $7 \%$, one expects that, strictly speaking, the Frank-Van der Merwe growth mode cannot be realized for this system. Nevertheless, 3D island formation is only observed in growth on the GaAs(001) substrate, while being absent on the other lowindex surfaces. This behavior has been ascribed to plastic relaxations in the films grown on the (110) and (111) surfaces of GaAs that reduce the elastic strain in the films grown on these substrates. ${ }^{8}$ Apparently, such a mechanism of strain relief is absent on $\mathrm{GaAs}(001)$, and the morphological features associated with Stranski-Krastanow growth show up in films grown on this substrate. For a long time, it was believed that 3D island growth in both the Volmer-Weber mode and Stranski-Krastanow mode is accompanied by plastic relaxation, for instance, by the formation of dislocations near the island base. In recent years, it has been found for several heteroepitaxial systems that dislocation-free (called coherent) islands form in Stranski-Krastanow growth. ${ }^{9-14}$ These islands relieve much of the strain-induced elastic energy by changing the in-plane lattice constant in the island for layers away from the interface. These nanoscale coherent islands, which are often found to have a very narrow size distribution and to be arranged in a regular array, ${ }^{11-13}$ are promising to be used in the quantum dot light emitting diodes (LEDs) and lasers. However, the mechanism of formation of coherent islands in highly lattice-mismatched heteroepitaxy is not understood. In fact, the discussion is highly controversial and more theoretical effort is clearly needed.

From a thermal-equilibrium picture, it is understood that forming 3D coherent islands in Stranski-Krastanow growth is energetically more favorable compared to a uniformly strained film because the gain of elastic relaxation energy in an island overcompensates the cost due to the increased surface energy by islanding. Naturally, one may attribute the observed islands with the narrow size distribution to a minimum of the free energy of the system. However, an equilibrium theory with only two energetic contributions, a positive 
one from the island surface energy $\left(E \sim V^{2 / 3}, V\right.$ is the island volume) and a negative one from the elastic relaxation energy $(E \sim V)$, fails to predict a finite equilibrium size of the islands. ${ }^{15,16}$ In order to cope with this difficulty, several additional effects, e.g., a negative surface energy contribution by taking into account nonlinear terms of surface strain and a contribution from interactions between islands, ${ }^{17,18}$ have been invoked. Priester and Lannoo ${ }^{15}$ proposed a mechanism in which 2D platelets act as precursors for the formation of 3D coherent islands, thus determining their size. However, it is shown experimentally ${ }^{19}$ that the surface density of the platelets just before the 3D transition and the island density just after the 3D transition are quite different, and that the distribution of 2D platelets and 3D islands on the surface also appears to be strongly different. Thus, the platelets cannot be considered as the direct precursors of the (much larger) 3D islands, but some intermediate processes occur between platelet formation and the appearance of fully faceted islands. Most recently, the observation of island ripening ${ }^{20}$ has also made it doubtful if the islands can be interpreted at all as structures in total equilibrium.

We previously showed that the size of the coherent islands can be understood as the result the energetic balance that governs material transport between the wetting layer and the islands. ${ }^{21}$ This situation corresponds to an equilibrium state with an additional constraint that the island density is fixed. In this sense, we extend the notion of StranskiKrastanow growth to a situation where the system does not reach full thermodynamic equilibrium, which would correspond to one single large island, but still a local equilibrium between the various facets of an island and the surrounding wetting layer is achieved. The previous study also indicated that the wetting layer contribution plays an important role for this particular growth mode. In fact, both experimental and theoretical investigations ${ }^{12,22}$ had shown that the thickness of the wetting layer is reduced after the transition to 3D surface morphology, i.e., the growing islands are fed by atoms from the wetting layer. This process is energetically favorable in the initial stage. However, after a certain amount of material is assembled into islands, further incorporation of atoms from the wetting layer would result in an unfavorable thinning of the wetting layer. In turn, the surface energy of the wetting layer and the system energy are raised. Thus, for given values of the nominal film thickness and the island density, the islands have an optimum size. This explanation for the island size by the constrained equilibrium theory is in accord with the recent observation ${ }^{20}$ of island ripening.

In this study, we aim at showing that both a well-defined island size and shape can be obtained under these conditions. To make the discussion more transparent, we choose a fairly simple type of islands, truncated InAs pyramids with $\{110\}$ side facets (see Fig. 1). In this case the shape can be described by a single parameter, the aspect ratio $h / a$, and we will determine its dependence on the island volume. Our results show that there is a higher value of the height-to-base ratio for a larger island, and this results in an enhanced elastic energy relaxation. ${ }^{24}$ However, we will also present results for other shapes for comparison, namely, for pyramidal islands used earlier, ${ }^{21}$ and for islands with the optimized shape consisting of $\{110\},\{001\},\{111\}$, and $\{\overline{1} \overline{1} \overline{1}\}$ facets. $^{23}$
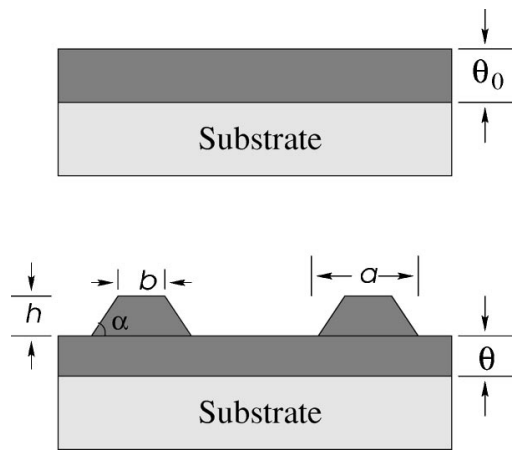

FIG. 1. Schematic illustration of the formation of coherent islands on the substrate surface. $\theta_{0}$ is the nominal coverage (i.e., the total amount of deposited material) and $\theta$ is wetting layer thickness. $\alpha$ is the tilt angle of island facets. The square island base corresponds to the [110] and [ $\overline{1} 10]$ directions and the bottom face and top face are $a^{2}$ and $b^{2}$, respectively. $h$ is the island height.

The present paper is organized as follows. In Sec. II, we define the energy contributions and present our parameterfree theoretical model. We calculate the surface energies (for both the island facets and the wetting layer) using densityfunctional theory within the local-density approximation with the help of the plane-wave pseudopotential method. ${ }^{25}$ The elastic energy in both the islands and the substrate is calculated within continuum elasticity theory. In Sec. III, we present the results and discussions. The theoretical results are also compared with experiments. We conclude in Sec. IV.

\section{TOTAL ENERGY GAIN, SURFACE ENERGY, AND ELASTIC RELAXATION ENERGY}

We propose a view of the quantum dot growth process divided into three phases: an early nucleation phase which mainly determines the island density $n$, a second phase where the islands grow mostly on expense of the wetting layer, sometimes, depending on the growth conditions, followed by a third phase characterized by Ostwald ripening. As long as the wetting layer acts as a source for material, existing nuclei will grow rapidly. Hereby the island density $n$ remains constant ${ }^{12}$ during the growth. We treat it as an input to our model noting that it may be determined separately by a theory of the growth kinetics. ${ }^{26,27}$ We consider an ensemble of islands with an area density $n$ and average volume $V$. After deposition is completed, the amount of material on the surface is conserved, and it may arrange itself either in a smooth film, or in islands on a film which is correspondingly thinner. We define the energy gain due to islanding as the energy difference between the film with islands (see the lower part of Fig. 1) and a homogeneous film containing the same amount of material (see the upper part of Fig. 1). For the maximum attainable island size, the relevant quantity is the energy gain per unit island volume. A minimum of this quantity indicates the optimum island size, where the islands stop to grow, since further addition of atoms would no longer be energetically favorable. While it is difficult to evaluate the total energy of an island with several thousand atoms by a direct density functional calculation, a separation of the energy into surface, bulk, and other contributions has been proven to be valid. ${ }^{15,28,29}$ The energy gain per unit volume due to islanding is 


$$
E_{\text {tot }} / V=E_{\text {relax }} / V+E_{\text {surf }} / V+E_{w l} / V
$$

where $E_{\text {tot }}$ is the total energy gain of an island with the volume $V . E_{\text {relax }}$ is the difference in elastic energy in the situation with islands, compared to a homogeneous film with the same amount of material. $E_{\text {surf }}$ is the energy of the additional surface generated by island formation. $E_{w l}$ is the contribution by the thinning of the wetting layer that goes along with island formation. In Eq. (1), we omit the repulsive interaction between islands due to their induced stress fields. The interaction between islands is not important for a dilute or moderate density system. ${ }^{15,30} \mathrm{We}$ omit as well the edge and corner energies which do not have an important influence on the calculated results for large islands. ${ }^{15,23,28,29}$ The entropic contributions to the free energy are also neglected, assuming that the entropic contributions before and after the 3D transformation are very similar. We evaluate the contributions to Eq. (1) using a previously developed hybrid method based on $a b$ initio calculations and elasticity theory. ${ }^{23}$

To describe the driving force for 3D island formation, the elastic relaxation of material in the islands, we introduce the elastic relaxation energy, which is the elastic energy difference between the film with islands (see lower part of Fig. 1) and a homogeneous, uniformly strained film (see upper part of Fig. 1), as

$$
E_{\text {relax }} / V=E_{\text {elast }}^{\text {is }} / V-\epsilon_{\text {film }},
$$

where $E_{\text {elast }}^{\text {is }}$ is the island elastic energy and $\epsilon_{\text {film }}$ is the elastic energy density in a homogeneous, uniformly strained film, as obtained from elasticity theory. For a sufficiently thick film, it is fully justified to separate the formation energy of the film into the elastic, surface, and interface energies. However, such a distinction is no longer possible for ultrathin films of only a few MLs thickness. In the latter case, the term $\epsilon_{\text {film }}$ in Eq. (2) should be regarded as a formal convention, and an $a b$ initio treatment of such strained films, in which all contributions are included automatically, is required (see below). As far as the side facets of the islands are concerned, we treat the contribution coming from intrinsic surface stress as part of the surface energy [see Eq. (3)]. A discussion of the validity of this procedure can be found in Ref. 28. For an island containing a sufficiently high number of atoms, e.g., 5000 atoms or more, it turns out that the strain fields and elastic energies are well described by continuum elasticity theory. ${ }^{15,23}$ Therefore the long-range strain relaxation in the island and in the underlying substrate is evaluated by elasticity theory applying a finite-element approach. ${ }^{23,24}$ The experimental elastic moduli are employed to describe the elastic properties of both the island and the substrate. The calculations are performed for a pyramidal shape island. For a truncated shape island (see Fig. 1), a scaling law is employed to obtain the elastic energy. ${ }^{24}$ Details of the calculations have been presented previously. ${ }^{23,24}$ The results obtained from elastic theory show that the elastic energy has a linear scaling relation with the volume for an island with a fixed shape, e.g., for the pyramidal shape island; but this is not true when the island shape depends on the volume. ${ }^{16}$

The surface energy increase by islanding is given by

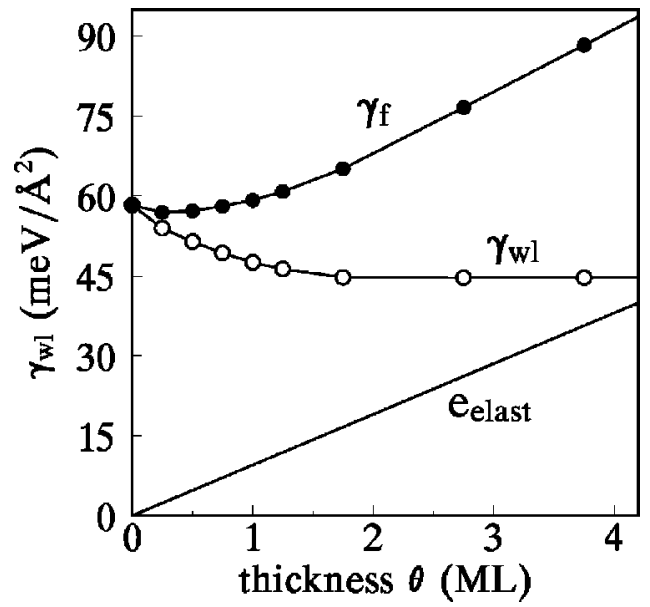

FIG. 2. Surface plus interface energy $\gamma_{w l}$ as a function of thickness $\theta . \gamma_{f}$ is the formation energy of the wetting layer per unit area. $e_{\text {elast }}$ is the elastic energy of the wetting layer for a volume of unit area $\times$ thickness $\theta \times L$. From various configurations with $N_{\text {In }}$ $=8 \theta \mathrm{In}$ atoms per $(2 \times 4)$ surface unit cell, those with the lowest formation energy are presented.

$$
E_{\text {surf }}=\sum_{i}\left(\gamma^{i}+\sum_{j k} \sigma_{j k}^{i} \bar{\varepsilon}_{j k}^{i}\right) A^{i}-\gamma_{w l}\left(\theta_{0}\right) A^{0},
$$

where $\gamma^{i}$ is the surface energy of an InAs island facet (unstrained) and $\gamma_{w l}\left(\theta_{0}\right)$ is the surface plus interface energy of the wetting layer with the nominal thickness $\theta_{0}$ (see below). $A^{i}$ and $A^{0}$ are the area of the $i$ th island facet and the island base area, respectively. $\sigma$ and $\bar{\varepsilon}$ are the island surface stress and strain averaged over the facet. In Eq. (3), we consider the surface stress contribution to the island surface energy up to the linear term.

The third term in Eq. (1) describes change in the formation energy of the wetting layer as a result of its thinning, and is defined as

$$
E_{w l}=\left(1 / n-A^{0}\right) \times\left[\gamma_{w l}(\theta)-\gamma_{w l}\left(\theta_{0}\right)\right] .
$$

$\gamma_{w l}(\theta)$ is defined as the surface energy of the wetting layer plus the energy associated with the interface between the film and the substrate. It is displayed as a function of the wetting layer thickness $\theta$ in Fig. 2. By definition, $\gamma_{w l}(0)$ corresponds to the surface energy of the substrate. For $\theta$ $<1 \mathrm{ML}$, the wetting layer consists of a surface alloy between InAs and GaAs, with $\theta$ being the concentration of In atoms in the topmost cation layer. As we did previously, ${ }^{21}$ we artificially decompose the total formation energy $\gamma_{f}$ of the wetting layer into the surface plus interface contribution $\gamma_{w l}$ and the elastic energy contribution $e_{\text {elast }}$ (see Fig. 2). $\epsilon_{\text {film }}$ in Eq. (2) is the elastic energy per unit volume in the wetting layer, and is equal to $e_{\text {elast }} /(\theta \times L)$. In this way, we keep the form of the first two terms in Eq. (1) the same as in earlier work. ${ }^{23}$ Again, one should keep in mind that the distinction between elastic energy, the surface and interface energies becomes unphysical for ultrathin films. However, the present treatment of subtracting the elastic energy from the formation energy of the wetting layer and putting it into the elastic energy contribution is only a matter of accounting and does not affect the total energy gain by islanding. The $a b$ initio calculations for the strained wetting layer take full account of 
TABLE I. Surface energies $\gamma$ and surface stresses $\sigma_{x}, \sigma_{y}$ for InAs surface reconstructions with the chemical potential $\mu_{\mathrm{As}}$ $=\mu_{\mathrm{As}(\text { bulk })}-0.2 \mathrm{eV}$. The blanks in the table mean the values are not calculated because the top surface of the island is strain free.

\begin{tabular}{lcccc}
\hline \hline surface & & $\begin{array}{c}\gamma \\
\left(\mathrm{meV} / \AA^{2}\right)\end{array}$ & $\begin{array}{c}\sigma_{x} \\
\left(\mathrm{meV} / \AA^{2}\right)\end{array}$ & $\begin{array}{c}\sigma_{y} \\
\left(\mathrm{meV} / \AA^{2}\right)\end{array}$ \\
\hline$(110)$ & cleavage & 41 & 26 & 54 \\
$(100)$ & $\beta 2(2 \times 4)$ & 47 & & \\
$(111)$ & In vacancy & 42 & 48 & 48 \\
$(\overline{1} \overline{1} \overline{1})$ & As trimer & 49 & 92 & 92 \\
\hline \hline
\end{tabular}

all energetic contributions even for thin films. We note that the final thickness of the wetting layer $\theta$ is related to the average volume of the islands $V$ by the law of mass conservation $V=(1 / n)\left(\theta_{0}-\theta\right) L$, where $L$ is the monolayer thickness.

In order to obtain accurate values for the surface energies, the formation energy of the wetting layer, and intrinsic surface stresses, we perform $a b$ initio total energy calculations within the framework of density-functional theory with the local-density approximation for the exchange-correlation energy functional. ${ }^{31} \mathrm{~A}$ slab including eight or nine atomic layers for the anion- or cation-terminated surfaces separated by a sufficiently large vacuum layer is used to represent the surfaces, and one side of the slab is passivated by charged $(Z=1.25) \mathrm{H}$ atoms. ${ }^{32} \mathrm{We}$ employ the fhi96md code ${ }^{25}$ and the norm-conserving, fully separable pseudopotentials. ${ }^{33-35}$ The wave functions are expanded into plane waves with the energy cutoff 10 Ry. The Brillouin zone integration of the electron density is performed using special k-point sets. ${ }^{36}$ The top six or seven atomic layers for the anion- or cationterminated surfaces are relaxed until the forces on the atoms are smaller than $25 \mathrm{meV} / \AA$. The formation energies of the surfaces are obtained by subtracting the calculated total energy of an appropriate amount of bulk material. In order to obtain the absolute surface energies for (111) and ( $\overline{1} \overline{1} \overline{1})$ orientations, the energy-density formalism introduced by Chetty and Martin ${ }^{37}$ is employed. Details of the calculations can be found in Ref. 38 and the results have been presented in Ref. 23.

A compound material such as InAs consists of two elements and thus the difference of the number of atoms of the two species enters as another degree of freedom in addition to the atomic geometry. Nonstoichiometric surfaces are considered by allowing the surface to exchange atoms with a reservoir, which is characterized by a chemical potential, in our case the arsenic chemical potential $\mu_{\mathrm{As}}$. Since epitaxial growth is mostly performed under As-rich conditions, we consider this chemical potential to be close to equilibrium with bulk arsenic (i.e., $\mu_{\mathrm{As}}=\mu_{\mathrm{As}(\mathrm{bulk})}-0.2 \mathrm{eV}$ ). For each facet, we have selected the reconstruction with the lowest energy from several candidates ${ }^{23}$ for the chemical potential under which the island growth is evaluated. The calculated results are given in Table I. Under the chemical potential $\mu_{\mathrm{As}}=\mu_{\mathrm{As}(\mathrm{bulk})}-0.2 \mathrm{eV}$, the $\mathrm{GaAs}(001)$ substrate has the $\beta 2(2 \times 4)$ reconstruction. For the wetting layer, we find that the $\beta 2(2 \times 4)$ reconstruction is the lowest energy reconstruction for various InAs coverages under this chemical potential. The atomic structure model of reconstructed InAs films

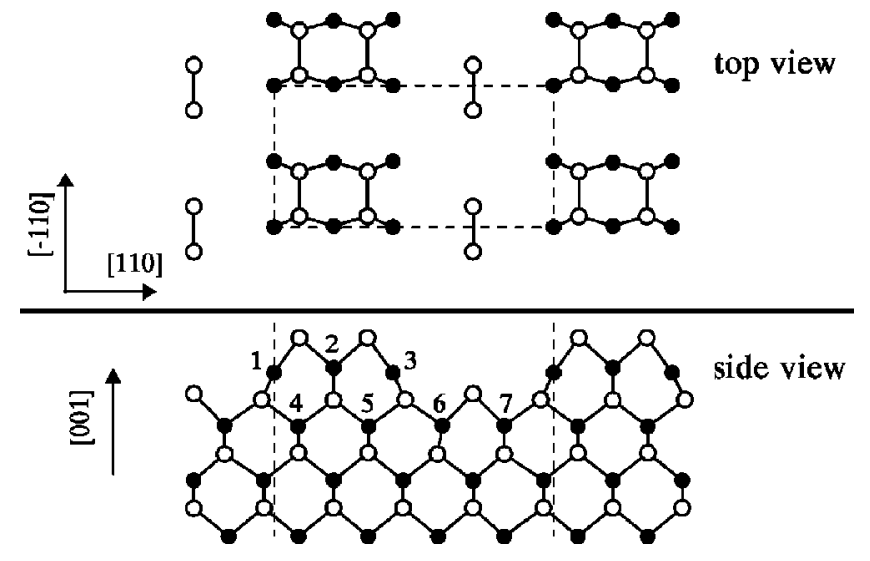

FIG. 3. The atomic structure model of the $\beta 2(2 \times 4)$ reconstruction. Filled and open circles denote cation and anion atoms, respectively. The supercell used in $a b$ initio calculations is indicated by dashed lines. The numbers denote the sites which In atoms may occupy to form a surface alloy for low InAs coverage cases.

is shown in Fig. 3. In our study, we consider a number of atomic configurations. Each number in Fig. 3 indicates a pair of sites that may either be occupied by Ga atoms or In atoms for low coverage cases. Our results indicate that the In atoms are most favorably incorporated by substituting the Ga atoms in the topmost cation layer. Hence In atoms tend to segregate to the GaAs surface. For large InAs coverages, structures with an abrupt interface between the InAs layer and the substrate with the $(1 \times 1)$ periodicity have lowest energies. For example, at $0.75 \mathrm{ML}$ InAs coverage, six In atoms occupy the pairs of sites 1,2, and 3, while eight In atoms (corresponding to 1 ML InAs coverage) occupying the sites 1, 2, 3, and 6 have the lowest energy. $14 \mathrm{In}$ atoms (corresponding to 1.75 ML InAs) tend to occupy the pairs of sites 1-7. We also consider several other reconstructions, such as the $\alpha(2 \times 4)$ and $\beta(2 \times 4)$ reconstructions formed by filling the trenches of $\beta 2(2 \times 4)$ by In adatoms or by both In and As atoms. ${ }^{39}$ In Fig. 2, we show the calculated results of the surface plus interface energy of the wetting layer for various InAs coverages. Only those configurations with the lowest formation energy for various InAs coverages are presented in Fig. 2. We can see from Fig. 2 that the surface plus interface energy of the wetting layer becomes less dependent on $\theta$ when it is larger than 1.75 ML. Experiments observed the $(2 \times 4)$ reconstruction in between islands. ${ }^{40,41}$ Different explanations for this observation have been offered: In one study, the ( 2 $\times 4)$ reconstruction of the wetting layer was explained as the surface structure of the exposed GaAs substrate, because the deposited InAs would be transferred to the newly formed islands $;{ }^{40}$ another explanation for the $(2 \times 4)$ reconstruction of the wetting layer is that the wetting layer is sufficiently thick and, therefore has the pure InAs surface reconstruction. ${ }^{23,42}$ Using the scanning tunneling microscopy (STM), Belk et al. ${ }^{43}$ found that the wetting layer may have the $(1 \times 3)$ or $(2 \times 3)$ reconstruction resulting from alloying; but they also reported that it is the $(2 \times 4)$ reconstruction when the InAs layer is thicker than 1.2 ML. In other STM work, a $(4 \times 4)$ or $c(4 \times 4)$ In-terminated reconstruction was also distinguished from the $(2 \times 4) \mathrm{GaAs}$ substrate in the initial growth stages. ${ }^{44}$ 


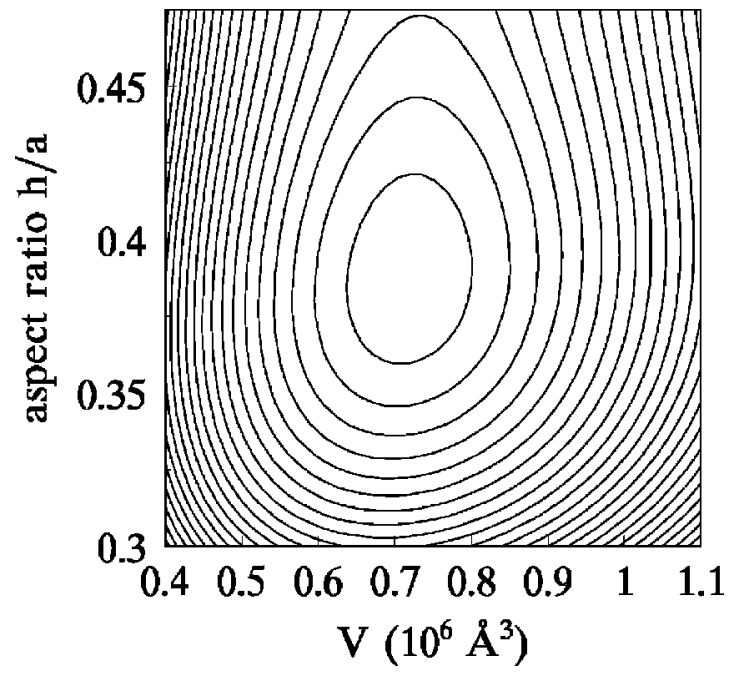

FIG. 4. Contour plot of the energy gain for $n=10^{10} \mathrm{~cm}^{-2}, \theta_{0}$ $=1.8 \mathrm{ML}$. The contour spacings are $0.0025 \mathrm{meV} / \AA^{3}$.

\section{RESULTS AND DISCUSSIONS}

\section{A. Island size and shape}

The approach taken in this paper allows us to simultaneously optimize the size of the islands and their aspect ratio. In Fig. 4, we show the total energy gain per unit volume of the truncated pyramidal island depending on the island volume $V$ and aspect ratio $h / a$ for $n=10^{10} \mathrm{~cm}^{-2}, \theta_{0}=1.8 \mathrm{ML}$. The contour spacings are $0.0025 \mathrm{meV} / \AA^{3}$. This figure shows the optimum island size and aspect ratio obtained for the given island density and coverage. The minimum in Fig. 4 corresponds to an island with about 33000 atoms, which compares reasonably well with typical experimental values [between 20000 (Ref. 12) and 50000 atoms per island ${ }^{45}$ ].

Further, we perform a minimization of the total energy for several pairs of input parameters $\left(n, \theta_{0}\right)$, thus obtaining the optimum island volume and aspect ratio for each such pair. In Fig. 5, we show the aspect ratio as a function of island volume. We can see that the island shape varies when the island volume increases, but little dependence on the island

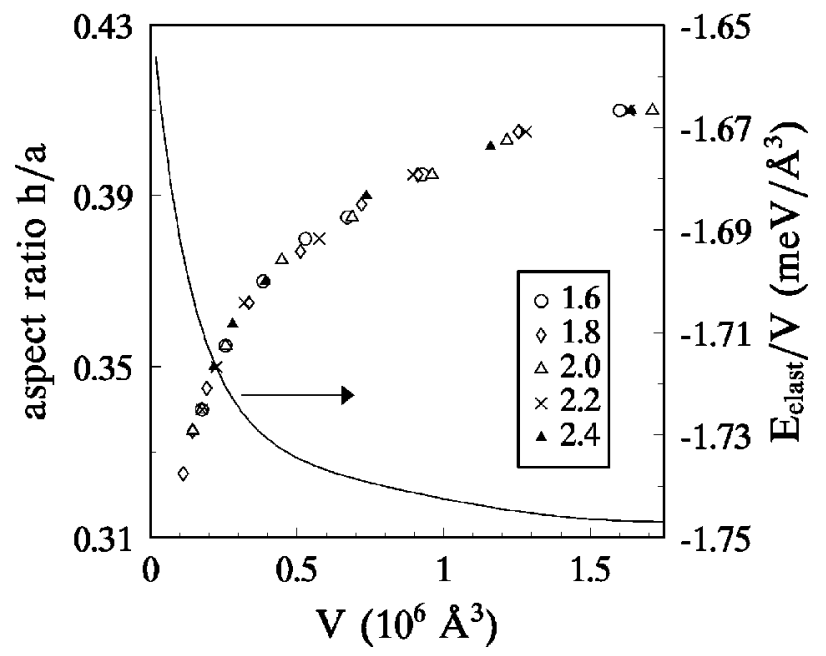

FIG. 5. Dependence of the island aspect ratio and the elastic relaxation energy (solid line) on the island volume. The symbols correspond to different coverages as indicated in the figure.

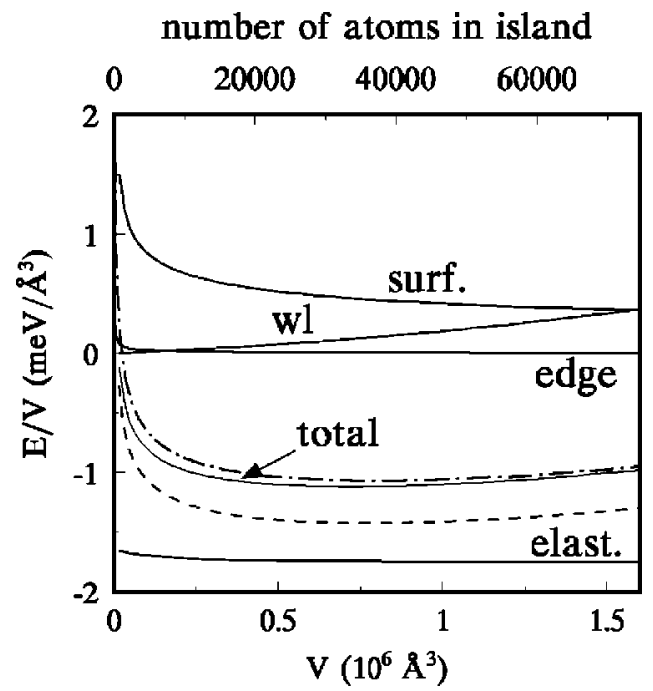

FIG. 6. Dependence of total energy gain and various energy contributions (solid lines) on the island volume or the number of atoms (upper scale) for $n=10^{10} \mathrm{~cm}^{-2}, \theta_{0}=1.8 \mathrm{ML}$. The total energy gains for the pyramidal island (dash-dotted line) and for the island with the optimum shape (dashed line) are also shown.

density and the nominal coverage is found. The relation between island shape and island volume predicted earlier ${ }^{48,49}$ has also been observed by experiments. ${ }^{46,47}$ Because the elastic energy relief per unit volume is determined only by the island shape, the present results indicate that an island adjusts its shape to relieve the elastic energy as much as possible, and the final island shape is determined by minimizing the sum of the elastic relaxation energy and the island surface energy. Thus energetics plays a key role in island growth and some previous studies based on energetics had predicted some important aspects of island growth, such as the critical layer thickness, ${ }^{22,21}$ island size, ${ }^{15,21,50,51}$ island shape ${ }^{23,24,48,49}$ and stability. ${ }^{17,18,21}$ Since the islands have a narrow size distribution, we can predict that these islands must also have a very similar aspect ratio, especially for the islands having a larger volume (see Fig. 5). The calculated aspect ratio for the experimental island size (between 20000 and 50000 atoms per island) is between 0.37 and 0.40 . This is in agreement with most recent experimental observations that the island with the $\{110\}$ facets has an aspect ratio of 0.33 . ${ }^{47}$ Some earlier experiments obtained much lower aspect ratio values 0.25 (Ref. 11) and $0.13 .^{12}$ From the experimental island aspect ratio, it has been concluded that some high index faceted planes, such as $\{311\},\{401\}$, or $\{511\}$, etc., should be present. ${ }^{12,53}$ This is in line with RHEED observations, where fractional index diffraction peaks were observed ${ }^{54-56}$ However, steeper islands with low index facets, such as $\{110\}$ side facets, are expected to have a higher aspect ratio. ${ }^{52,47}$ Thus the sometimes observed lower aspect ratio may be an indication that equilibrium with respect to island shape is not always reached in the experiments, but kinetics may have an impact on the island shape as well.

We show in Fig. 6 the various energy contributions and the total energy gain per unit volume for $n=10^{10} \mathrm{~cm}^{-2}, \theta_{0}$ $=1.8 \mathrm{ML}$. The total energy gain is decomposed into the contributions in Eq. (1). The elastic relaxation energy [the first term in Eq. (1) and defined in Eq. (2)] is negative due to strain relief. It is noticeable that the elastic relaxation energy 


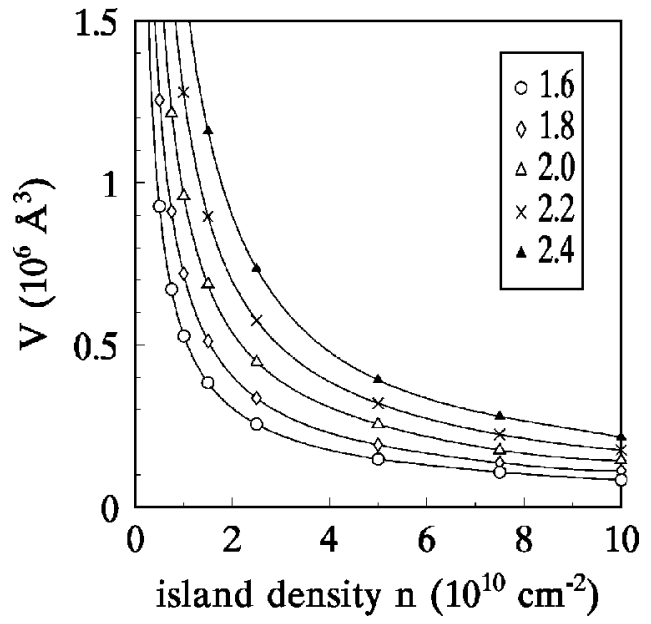

FIG. 7. Dependence of the optimum island size on the island density and the nominal coverage. The latter, i.e., $\theta_{0}$, ranges from 1.6 to 2.4 monolayers.

no longer linearly depends on the island volume after we take shape variations into account. In Figs. 5 and 6, we show the dependence of the elastic relaxation energy on the island volume. We can see larger islands relieve more elastic energy per unit volume due to the higher value of the heightto-base ratio. The surface energy contribution [the second term in Eq. (1) and defined in Eq. (3)] is a cost, and therefore it is positive. It is proportional to the island surface area. The wetting layer energy contribution [the third term in Eq. 1 and defined in Eq. (4)] is also positive and depends complexly on the island volume, island density, and coverage. We also show the energy contribution of the edges in Fig. 6, which becomes negligible compared to the other contributions for large islands (estimated as in Ref. 23). The contribution by the wetting layer is crucial for obtaining the minimum of the total energy gain curve, which corresponds to the existence of an optimum island size.

In Fig. 6, we also present the results for the pyramidal island and the island with the optimum island shape consisting of the low index facets $\{110\},\{001\},\{111\}$, and $\{\overline{1} \overline{1} \overline{1}\}$ as discussed in Ref. 23. Generally, we find the energy gain to increase if we allow for more freedom in the island shape, going from a full pyramid, to a truncated pyramid, to more complex shapes (see Fig. 6). However, the total energy gain curves for various island shapes behave similarly. This indicates that employing a simple island shape, which does not even include the island shape change as the volume varies, for example, a pyramidal island, does not prevent us from capturing the important features of island growth. The optimum island size predicted by employing the different island shapes is only slightly affected.

\section{B. Dependence of the island size on the island density and coverage}

Figure 7 shows the results that the optimum island size depends on the density and coverage. The island volume is a hyperbolic function of island density $n$ for a constant coverage. For a larger island density, the optimum island size is smaller. However, the relation is more complicated than solely expected from mass conservation. In fact, the total

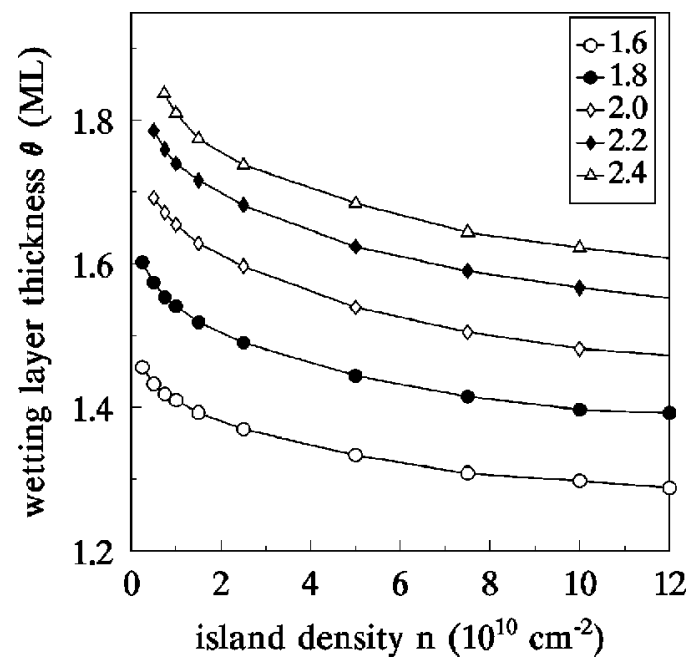

FIG. 8. Dependence of the wetting layer thickness on the island density and the nominal coverage.

material in islands will also depend on the island density $n$ for a given coverage. We have investigated the ratio of the material in islands to the total deposition, and find that only about $10-30 \%$ of the total deposited material is assembled into islands, depending on the nominal coverage and the island density. Our results show that there always exists a wetting layer thicker than $1 \mathrm{ML}$ after the 3D transformation, its thickness depending on the island density and the nominal coverage (see Fig. 8). The previous view that the $(2 \times 4)$ reconstruction of the wetting layer is due to the exposition of the substrate GaAs surface after the 3D transformation ${ }^{40}$ is incorrect according to the present calculations. A crosssectional scanning-tunneling microscopy investigation also indicated the existence of a wetting layer which is thicker than $1 \mathrm{ML}^{57}$

Experimentally, ${ }^{58,59}$ the island size is found to vary with the growth temperature. This was attributed to a kinetically limited process, ${ }^{58,59}$ consistent with the theory presented here, since the island density is known to depend strongly on the growth temperature, ${ }^{26,27}$ and thus kinetics controls the growth through controlling the island nucleation density. Figure 7 also shows that the island size increases with the increase of nominal coverage for a constant density. In Fig. 9 , we show that our theory can reproduce very well the increase of the island half-base with the amount of deposited material observed experimentally, by using a suitable island density as input. The island density $1.6 \times 10^{10} \mathrm{~cm}^{-2}$ used to fit the experimental results ${ }^{60}$ also agrees very well with the experimentally estimated island density $1.5-2 \times 10^{10} \mathrm{~cm}^{-2}$.

Our results also show that the transition to $3 \mathrm{D}$ growth is not only triggered by the amount of deposited material, but also by the sample preparation. For a given coverage, the 3D morphology transition will only occur if the island nucleation density is not too high. When the density of island embryos is larger than a certain density, e.g., $3.0 \times 10^{11} \mathrm{~cm}^{-2}$ for the coverage 1.6 ML, fully facetted 3D islands cannot stabilize themselves. In other words, the critical layer thickness at which the 3D islands start to form depends on the island density, i.e., growth conditions. It is thinner for the case with a lower island nucleation density, which may typically result from high substrate temperatures. Reversely the 


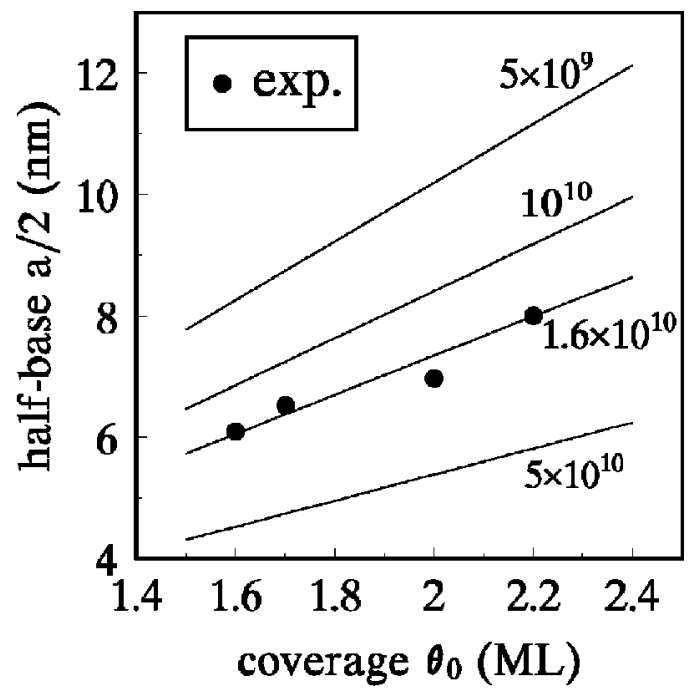

FIG. 9. Dependence of the island size on the nominal coverage. The full circles indicate the results obtained by the photoluminescence measurements in Ref. 60.

film will grow two dimensionally to a larger thickness before the $3 \mathrm{D}$ transformation in the case of having a large island nucleation density. ${ }^{21,61}$

\section{Long-time evolution of uncapped islands}

After deposition of new material has been stopped and after each individual island has reached its own local thermal equilibrium state with respect to its different facets and the wetting layer it is sitting on, exchange of atoms between neighboring islands may take place on a longer time scale. If we allow for variations of the island density in our theory, we find that larger islands in conjunction with a lower island density (see Fig. 7) are energetically preferred. The total energy gain per unit volume is shown in Fig. 10. Thus our theory not only explains the optimum island size in constrained equilibrium, but also accounts for Ostwald ripening, which proceeds by a steady (it may be very slow) decrease of the island density with time. Most recently, Lee et al. ${ }^{20}$ succeeded to observe experimentally the ripening of $\mathrm{CdSe}$ is-

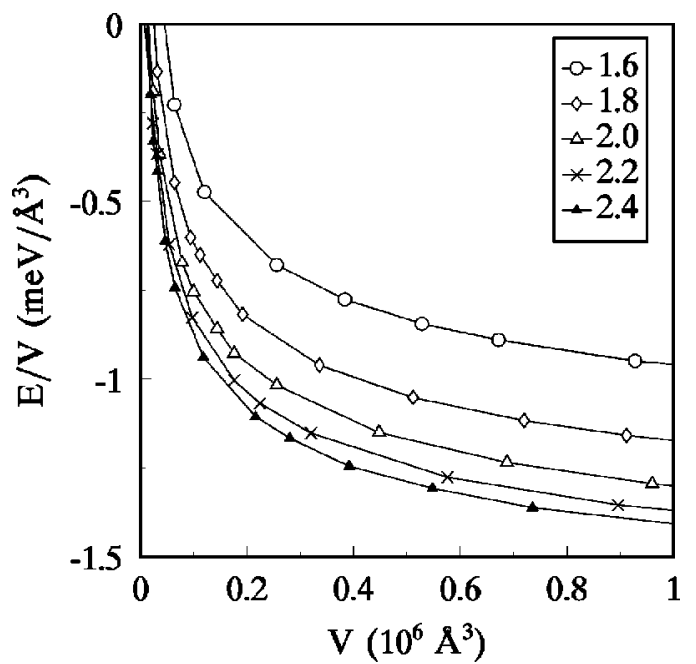

FIG. 10. Total energy gain as a function of island volume for various coverages. lands after preserving their samples at room temperature for several days. Generally, the time scale for ripening is set by material transport between islands on the substrate surface. Since this is an activated process, the material transport at room temperature is many orders of magnitude slower than at the growth temperature. For the arsenide compound semiconductors, one further has to keep in mind that surface mobility of chemisorbed As atoms is not required during growth, since the As supply is mediated by a weakly bound mobile precursor state of $\mathrm{As}_{2},{ }^{62}$ which is populated from the gas phase. In sharp contrast to growth, the material transport during ripening would require detachment and diffusion of both chemisorbed In and As species away from smaller islands, a process for which we would expect a sizeable energetic barrier. Since noticeable changes in the island size and density resulting from ripening typically take many days, ${ }^{20}$ the ripening is not an issue for device applications, where the islands are covered by a capping layer after a short growth interruption. Consequently, the ripening was not noticed in earlier experiments focussing on device fabrication.

From Fig. 10, we also see that the critical island size corresponding to zero energy gain is different for different coverages. As it is discussed above, the critical layer thickness depends on the growth conditions. ${ }^{21}$ The present results imply that for a different critical layer thickness the critical island size is also different, i.e., the critical island size needs to be larger for a thinner critical layer. This can be understood in terms of a larger energy barrier which must be overcome by the island embryo when it should grow on a thinner wetting layer. We can also see from Fig. 10 that incorporation of atoms from the wetting layer into islands from a thicker film yields a stronger energy relief than for a thinner film.

\section{CONCLUSIONS}

We presented a theoretical study of the formation of selfassembled coherent islands in the Stranski-Krastanow growth mode. We calculated the surface energies for both the island facets and the wetting layer using density-functional theory, and obtained the elastic energy in both the islands and the substrate from continuum elasticity theory. It is shown that an optimum island size exists for a given coverage and island density, if changes in the wetting layer morphology after the 3D transition are properly taken into account. This explains the narrow size distribution of quantum dots observed in some experiments, which is crucial for optoelectronic applications. Furthermore, we show how it is possible to attain a desired island size by properly choosing the growth conditions and the nominal coverage. Our theory reproduces the experimental trends observed in island growth well. Our study also indicates that the island shape (aspect ratio) only depends on the island size. However, the calculated total energy results also show that larger islands in conjunction with a lower island density are more stable, which means that the islands will undergo ripening but this will happen on considerably longer time scales.

\section{ACKNOWLEDGMENTS}

The authors thank E. Pehlke for fruitful discussions. This work was supported by Sfb 296 of the Deutsche Forschungsgemeinschaft. 
*Present address: Solid State Division, Oak Ridge National Laboratory, Oak Ridge, Tennessee 37831.

${ }^{1}$ F. C. Frank and J. H. Van der Merwe, Proc. R. Soc. London, Ser. A 198, 205 (1949).

${ }^{2}$ M. Volmer and A. Weber, Z. Phys. Chem. (Leipzig) 119, 277 (1926).

${ }^{3}$ I. N. Stranski and L. Krastanow, Sitzungsber. Akad. Wiss. Wien, Math.-Naturwiss. Klasse 146, 797 (1937).

${ }^{4}$ E. Bauer, Z. Kristallogr. 110, 372 (1958).

${ }^{5}$ Z. Y. Zhang and M. G. Lagally, Science 276, 377 (1997).

${ }^{6}$ G. Vidali, Surf. Rev. Lett. 4, 709 (1997); H. Röder, K. Bromann, H. Brune, and K. Kern, Surf. Sci. 376, 13 (1997).

${ }^{7}$ H. Brune, Surf. Sci. Rep. 31, 121 (1998).

${ }^{8}$ B. A. Joyce, T. S. Jones, and J. G. Belk, J. Vac. Sci. Technol. B 16, 2373 (1998).

${ }^{9}$ D. J. Eaglesham and M. Cerullo, Phys. Rev. Lett. 64, 1943 (1990).

${ }^{10}$ Y. W. Mo, D. E. Savage, B. S. Swartzentruber, and M. G. Lagally, Phys. Rev. Lett. 65, 1020 (1990).

${ }^{11}$ D. Leonard, K. Pond, and P. M. Petroff, Phys. Rev. B 50, 11687 (1994).

${ }^{12}$ J. M. Moison, F. Houzay, F. Barthe, L. Leprince, E. André, and O. Vatel, Appl. Phys. Lett. 64, 196 (1994).

${ }^{13}$ R. Heitz, T. R. Ramachandran, A. Kalburge, Q. Xie, I. Mukhametzhanov, P. Chen, and A. Madhukar, Phys. Rev. Lett. 78, 4071 (1997).

${ }^{14}$ N. Carlsson, W. Seifert, A. Petersson, P. Castrillo, M. E. Pistol, and L. Samuelson, Appl. Phys. Lett. 65, 3093 (1994).

${ }^{15}$ C. Priester and M. Lannoo, Phys. Rev. Lett. 75, 93 (1995), and references therein.

${ }^{16}$ N. Moll, Theorie der Form und Stabilität von Quantenpunkten auf III-V-Halbleitern (Wissenschaft und Technik Verlag, Berlin, 1998).

${ }^{17}$ V. A. Shchukin, N. N. Ledentsov, P. S. Kop'ev, and D. Bimberg, Phys. Rev. Lett. 75, 2968 (1995).

${ }^{18}$ I. Daruka and A. -L. Barabási, Phys. Rev. Lett. 79, 3708 (1997).

${ }^{19}$ M. Berti, A. V. Drigo, G. Rossetto, and G. Torzo, J. Vac. Sci. Technol. B 15, 1794 (1997).

${ }^{20}$ S. Lee, I. Daruka, C. S. Kim, A. -L. Barabási, J. L. Merz, and J. K. Furdyna, Phys. Rev. Lett. 81, 3479 (1998); S. H. Xin, P. D. Wang, Aie Yin, C. Kim, M. Dobrowolska, J. L. Merz, and J. K. Furdyna, Appl. Phys. Lett. 69, 3884 (1996).

${ }^{21}$ L. G. Wang, P. Kratzer, M. Scheffler, and N. Moll, Phys. Rev. Lett. 82, 4042 (1999).

${ }^{22}$ J. Tersoff, Phys. Rev. B 43, 9377 (1991).

${ }^{23}$ N. Moll, M. Scheffler, and E. Pehlke, Phys. Rev. B 58, 4566 (1998).

${ }^{24}$ E. Pehlke, N. Moll, A. Kley, and M. Scheffler, Appl. Phys. A: Solids Surf. 65, 525 (1997).

${ }^{25}$ M. Bockstedte, A. Kley, J. Neugebauer, and M. Scheffler, Comput. Phys. Commun. 107, 187 (1997); URL http://www.fhiberlin.mpg.de/th/fhimd

${ }^{26}$ J. G. Amar and F. Family, Phys. Rev. Lett. 74, 2066 (1995).

${ }^{27}$ H. T. Dobbs, D. D. Vvedensky, A. Zangwill, J. Johansson, N. Carlsson, and W. Seifert, Phys. Rev. Lett. 79, 897 (1997).

${ }^{28}$ J. Tersoff, Phys. Rev. Lett. 79, 4934 (1997).

${ }^{29}$ W. Yu and A. Madhukar, Phys. Rev. Lett. 79, 905 (1997); 79, 4939(E) (1997).

${ }^{30}$ A. Ponchet, D. Lacombe, L. Durand, D. Alquier, and J. -M. Cardonna, Appl. Phys. Lett. 72, 2984 (1998).
${ }^{31}$ J. P. Perdew and A. Zunger, Phys. Rev. B 23, 5048 (1981).

${ }^{32}$ K. Shiraishi, J. Phys. Soc. Jpn. 59, 3455 (1990).

${ }^{33}$ D. R. Hamann, Phys. Rev. B 40, 2980 (1989).

${ }^{34}$ L. Kleinman and D. M. Bylander, Phys. Rev. Lett. 48, 1425 (1982).

${ }^{35}$ M. Fuchs and M. Scheffler, Comput. Phys. Commun. 116, 1 (1999); URL http://www.fhi-berlin.mpg.de/th/fhimd

${ }^{36}$ H. J. Monkhorst and J. D. Pack, Phys. Rev. B 13, 5188 (1976).

${ }^{37}$ N. Chetty and R. M. Martin, Phys. Rev. B 45, 6089 (1992).

${ }^{38}$ N. Moll, A. Kley, E. Pehlke, and M. Scheffler, Phys. Rev. B 54, 8844 (1996).

${ }^{39}$ P. Kratzer, C. G. Morgan, and M. Scheffler, Phys. Rev. B 59, 15 246 (1999).

${ }^{40}$ N. Grandjean, J. Massies, and O. Tottereau, Phys. Rev. B 55, R10 189 (1997)

${ }^{41}$ S. Ohkouchi and N. Ikoma, Jpn. J. Appl. Phys., Part 1 34, L724 (1995).

${ }^{42}$ H. Yamaguchi and Y. Horikoshi, Phys. Rev. B 51, 9836 (1995).

${ }^{43}$ J. G. Belk, C. F. McConville, J. L. Sudijono, T. S. Jones, and B. A. Joyce, Surf. Sci. 387, 213 (1997).

${ }^{44}$ V. Bressler-Hill, A. Lorke, S. Varma, P. M. Petroff, K. Pond, and W. H. Weinberg, Phys. Rev. B 50, 8479 (1994).

${ }^{45}$ D. Leonard, M. Krishnamurthy, C. M. Reaves, S. P. Denbaars, and P. M. Petroff, Appl. Phys. Lett. 63, 3203 (1993).

${ }^{46}$ R. Leon, C. Lobo, J. Zou, T. Romeo, and D. J. H. Cockayne, Phys. Rev. Lett. 81, 2486 (1998).

${ }^{47}$ H. Saito, K. Nishi, and S. Sugou, Appl. Phys. Lett. 74, 1224 (1999).

${ }^{48}$ J. Tersoff and R. M. Tromp, Phys. Rev. Lett. 70, 2782 (1993).

${ }^{49}$ I. Daruka, J. Tersoff, and A. -L. Barabási, Phys. Rev. Lett. 82, 2753 (1999).

${ }^{50}$ Y. Chen and J. Washburn, Phys. Rev. Lett. 77, 4046 (1996).

${ }^{51}$ J. Tersoff and F. K. LeGoues, Phys. Rev. Lett. 72, 3570 (1994).

${ }^{52}$ D. Lacombe, A. Ponchet, S. Frechengues, V. Drouot, B. Lambert, and A. LeCorre, J. Cryst. Growth 202, 252 (1999).

${ }^{53}$ Y. Hasegawa, H. Kiyama, Q. K. Xue, and T. Sakurai, Appl. Phys. Lett. 72, 2265 (1998).

${ }^{54}$ H. Lee, W. Y. R. Lowe-Webb, and P. C. Sercel, Appl. Phys. Lett. 72, 812 (1998)

${ }^{55}$ Y. Nabetani, T. Ishikawa, S. Noda, and A. Sasaki, J. Appl. Phys. 76, 347 (1994).

${ }^{56} \mathrm{~T}$. S. Jones (private communication).

${ }^{57}$ H. Eisele, O. Flebbe, T. Kalka, and M. Dähne-Prietsch, Surf. Interface Anal. 27, 537 (1999); O. Flebbe, H. Eisele, T. Kalka, F. Heinrichsdorff, A. Krost, D. Bimberg, and M. DähnePrietsch, J. Vac. Sci. Technol. (to be published).

${ }^{58}$ G. S. Solomon, J. A. Trezza, and J. S. Harris, Jr., Appl. Phys. Lett. 66, 3203 (1995).

${ }^{59}$ D. E. Jesson, G. Chen, K. M. Chen, and S. J. Pennycook, Phys. Rev. Lett. 80, 5156 (1998).

${ }^{60}$ A. Polimeni, A. Patane, M. Capizzi, F. Martelli, L. Nasi, and G. Salviati, Phys. Rev. B 53, R4213 (1996), and references therein. We take the experimental values for the coverage thicker than $1.5 \mathrm{ML}$.

${ }^{61}$ C. Y. Snyder, J. F. Mansfield, and B. G. Orr, Phys. Rev. B 46, 9551 (1992).

${ }^{62}$ C. G. Morgan, P. Kratzer, and M. Scheffler, Phys. Rev. Lett. 82, 4886 (1999). 